
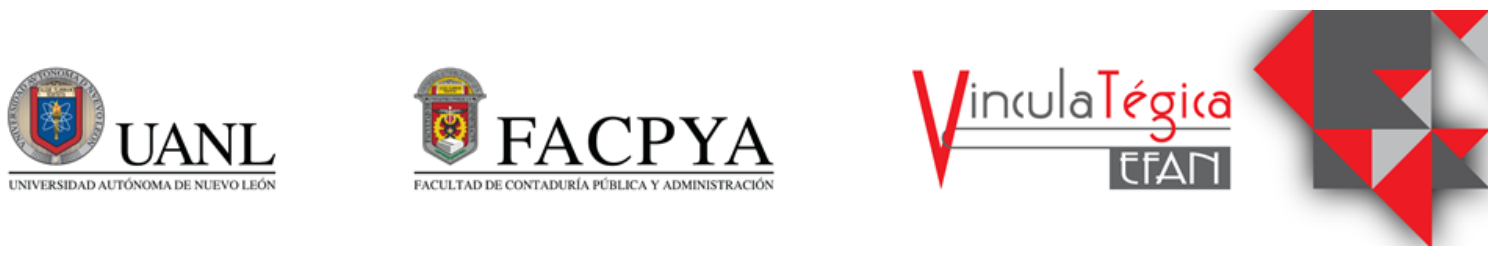

\title{
Aceptación y uso de la tecnología en profesores universitarios
}

\author{
Julymar Alegre Ortiz ${ }^{1}$, Ana Irene Cuevas Gutiérrez ${ }^{2}$ y Martha Magdalena Mendoza Vargas ${ }^{3}$ \\ ${ }^{1}$ Universidad Autónoma de Nuevo León, julymar.alegreort@uanl.edu.mx, Ciudad Universitaria, Pedro de Alba s/n \\ San Nicolás de los Garza, Nuevo León C.P. 66451 ,Tel. 81-2939-0507 \\ ${ }^{2}$ Universidad Autónoma de Nuevo León, acuevasg@uanl.edu.mx, Ciudad Universitaria, Pedro de Alba s/n San \\ Nicolás de los Garza, Nuevo León C.P. 66451,Tel. 81-1177-9077 \\ ${ }^{3}$ Universidad Autónoma de Nuevo León, martha.mendozavrg@uanl.edu.mx, Ciudad Universitaria, Pedro de Alba \\ s/n San Nicolás de los Garza, Nuevo León C.P. 66451,Tel .81-2647-0602
}

Información del artículo revisado por pares

Fecha de aceptación: junio-2021

Fecha de publicación en línea: diciembre-2021

DOI: https://doi.org/10.29105/vtga7.1-86

\section{Resumen}

El giro que la educación ha tomado a partir de la pandemia, hizo necesario que se plantearan nuevas alternativas para dar seguimiento a los programas educativo. La respuesta de las Instituciones de Educación Superior fue priorizar el uso de las Tecnologías de la Información y Comunicación por medio de actividades en línea, convirtiéndose en tarea de los profesores universitarios adaptarse a este nuevo proceso; esto nos lleva a la necesidad de entender cómo se sienten al respecto. El objetivo de este artículo, es analizar la aceptación y uso de la tecnología en profesores universitarios, considerando la antigüedad o el género como factores determinantes. A partir de una metodología de corte cuantitativo, con un diseño no experimental de alcance descriptivo y exploratorio, se utilizó la Teoría Unificada de Aceptación y Uso de la Tecnología (UTAUT) de Venkatesh et al., (2003), aplicando el cuestionario a 100 docentes. Los resultados permiten observar que los profesores universitarios muestran una aceptación positiva ante el uso de las tecnologías, pero si presentan Ansiedad, esto se comparó con la experiencia docente, encontrando que los profesores con más de 20 años de experiencia, reportaron valores más altos. Esto nos podría decir que, aunque se cuenta con soporte social e institucional, las dificultades ante el uso de

\section{Abstract}

The turn that education has taken since the pandemic, made it necessary to consider new alternatives to monitor educational programs. The response of Higher Education Institutions was to prioritize the use of Information and Communication Technologies through online activities, making it the task of university professors to adapt to this new process; This brings us to the need to understand how they feel in respect to it. The objective of this article is to analyze acceptance as well as usage of technology by university professors, considering seniority or gender as determining factors. Based on a quantitative methodology, with a nonexperimental design of descriptive and exploratory scope, the "Unified Theory of Acceptance and Use of Technology (UTAUT)" by Venkatesh et al. (2003) was used by applying the questionnaire to 100 teachers. The results allow us to observe that university professors show a positive acceptance of the use of technologies, but in some cases where they might present anxiety, this was compared with their teaching experience, finding that professors with more than 20 years of experience, reported higher values. This could tell us that, although there is social and institutional support, difficulties in the 
la tecnología, podrían marcar la diferencia de usarlas o no. Aunque esta investigación es exploratoria, se sugiere considerar más variables y una muestra más alta para analizar si se repite esta tendencia.

Palabras clave: aceptación de la tecnología, Educación Virtual, Modelo UTAUT, profesores universitarios.

\section{Introducción}

Sin duda alguna el giro que la educación ha tomado después del 11 de marzo del 2019 al ser declarada una pandemia por el Covid19 (OMS, 2020) ha arrojado transformaciones y retos en este sector. México no fue la excepción, y aunado a la decisión del gobierno de establecer un distanciamiento social, tuvo como consecuencia el impedimento de llevar a cabo clases presenciales en las instituciones educativas. Esto hizo necesario que se plantearán nuevas alternativas para dar seguimiento a los Programas Educativos.

Como respuesta, las Instituciones de Educación Superior agrupadas en la Asociación Nacional de Universidades e Instituciones de Educación Superior (ANUIES), presentaron acciones y estrategias para enfrentar esta contingencia de salud (ANUIES, 202), las cuales, entre sus opciones, priorizan el uso de las Tecnologías de la Información y la Comunicación como base de dichas estrategias.

De un momento a otro, los programas educativos cambiaron de ser presenciales a llevarse en línea, teniendo que adaptarse los contenidos casi al instante, de igual manera, se tuvo que capacitar al personal académico de manera intensa. Los docentes tuvieron que enfrentarse a retos pedagógicos y de socialización, la ruptura del esquema de revisar y casi auditar el progreso de sus estudiantes (Marione et al., 2020), además de enfrentarse a ellos que, al igual que los profesores, fueron volcados a un nuevo sistema de aprendizaje. Este contexto puso en evidencia la necesidad de estudiar cuál ha sido la reacción de los docentes universitarios ante este nuevo entorno inundado de tecnología, además de conocer como están siendo utilizados los recursos digitales a los use of technology could make a difference on whether or not to use them. Although this research is exploratory, it is suggested to consider more variables and a higher sample to analyze if this tendency is repeated.

Keywords: Technology Acceptance, Virtual Education, Model UTAUT, University Professors.

cuales tienen alcance. La aceptación a la tecnología en contextos educativos se ha enfrentado a 3 situaciones (Sánchez et al, 2017):

a. Barreras a la tecnología: Por ejemplo, problemas de conectividad $\mathrm{o}$ compatibilidad

b. Cuestiones relacionadas directamente con los alumnos: Distracción o brecha generacional

c. Factores relacionados con los docentes: Como la aceptación o adaptación de materiales.

Aunque estas tres situaciones están relacionadas, es en este último punto en el cual está enfocada la presente investigación, ya que los docentes son los que se encuentran guiando el proceso de construcción y conocimiento de los aprendizajes de sus estudiantes, ante el uso, casi de emergencia de las tecnologías educativas, las cuales han transformado el entorno académico, por lo cual es necesario analizar sus acciones y buenas prácticas para un mejor aprovechamiento.

Dentro del contexto global, la estrategia para poder enfrentar la crisis sanitaria ante la pandemia causada por el Covid19, acelero una situación que se sabía iba a ocurrir tarde o temprano, es decir, la integración de las tecnologías y la educación digital en los salones de clases ante la imposibilidad de que profesores y alumnos estuvieran trabajando de forma presencial (El Besomey, 2020).

El uso de la tecnología como estrategia para continuar los procesos de enseñanza y aprendizaje requirió que los profesores universitarios aceptaran su uso dentro de su práctica docente, lo que requiere de una formación virtual y una capacitación para su 
correcto manejo y utilización, pero esto a su vez nos lleva a la necesidad de entender cómo se sienten los profesores ante estas nuevas formas de trabajo.

Ante estas circunstancias, el objetivo central de este trabajo de investigación exploratoria consiste en analizar la aceptación y uso de la tecnología en profesores universitarios, conocer si la antigüedad como docente y el sexo de los mismos son factores de determinantes, esto a partir de la Unified Theory of Acceptance and Use of Technology (Teoría Unificada de Aceptación y Uso de la Tecnología, en adelante UTAUT) propuesto por Venkatesh et al., en el 2003, como un modelo desarrollado para probar la adopción y aceptación de la tecnología dentro del entorno educativo.

Conocer la adopción, aceptación y comportamiento sobre el uso de los sistemas tecnológicos de información no es algo reciente, ya que desde la década de los 70's, estos elementos eran estudiados como parte de las actividades para poder evaluar la calidad del software. Actualmente, esto se convierte en un requisito para la mayoría de los sistemas tecnológicos de información (Sivathanu \& Pillai, 2019), ya que se vuelve necesario tener diferentes métodos y modelos que expliquen la comprensión de los usuarios ante estos recursos, ya que factores como el uso, complejidad e incluso influencia social pueden afectar la decisión de seguir usándolos o no.

El uso que el usuario le dé al sistema tecnológico de información estará basado implícitamente en la intención que tenga de usarlo, y esto se relaciona con las creencias que tenga al respecto, por lo cual, la aceptación de estas herramientas se relaciona con la satisfacción ante su uso. Eso lo podemos ver en la Figura 1, propuesta por Momani (2018).

Figura 1. Influencia de la aceptación y satisfacción en la intención del uso continuo:

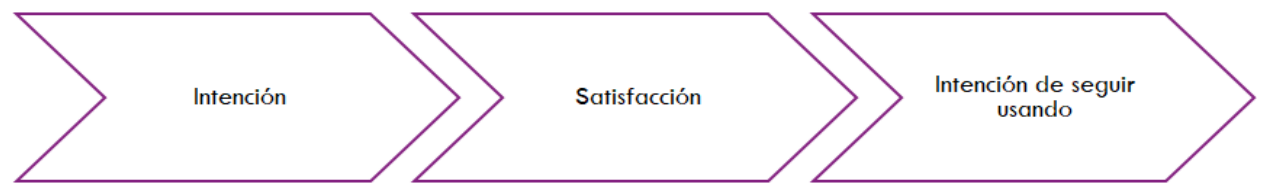

Fuente: Momani, 2018

\subsection{Antecedentes del Modelo UTAUT} 1.1.1. Aceptación y uso de la tecnología Modelos como este, intentaron explicar los diferentes factores existentes para que los usuarios tuvieran percepciones positivas ante el uso de herramientas tecnológicas como formación, experiencias, habilidades, conocimientos previos, etc.

Es a partir de las teorías de aprobación frente al uso de las tecnologías que se empezó a considerar los aspectos cognitivos y comportamentales (como las concepciones y percepciones, así como las actitudes), para explicar las conductas orientadas al uso favorable de estos recursos, donde el comportamiento exitoso no solo depende de la intención favorable, sino también del control de comportamiento (Cacante Caballero, 2020; Momani, 2020).

Esto se basa en el supuesto de un ser humano racional cuyos actos se encuentran influenciados por las experiencias que posee, y estas experiencias determinan la intención que los usuarios tendrán ante el uso y la implementación de la tecnología.

Diversos factores se han relacionado al momento de aceptar (e incorporar) el uso de las tecnologías, aunque la mayoría coinciden en ciertos factores, los cuales son la intención tanto la individual como la social. Esto da pie a la Teoría de Acción Razonada (TAR), propuesto originalmente por Fishbein y Ajzen (1980).

La TAR desde una perspectiva psicológica social intentó explicar los comportamientos individuales respecto al uso que se le daba a la tecnología, donde el comportamiento se verá influenciado por la intención conductual que se tenga (Alshammari \& Rosli, 2020), asumiendo entonces que las personas tomamos decisiones racionales, ya que estas son evaluadas continuamente, y a partir de los resultados que se obtienen, se forman las actitudes comportamentales (por ejemplo, el incorporar algo diferente o continuar usando determinada herramienta para la docencia). 
Este modelo es usado usualmente para explicar para explicar el comportamiento de las personas y su uso de la tecnología, debido al manejo de 3 componentes cognitivos: actitudes, normas sociales e intenciones, sin embargo, la desventaja de este modelo es que deja de lado el rol de hábito y las intenciones subjetivas (Taherdoost, 2018) por lo cual fue necesario generar modelos más explicativos.

\subsubsection{Estructura y Desarrollo de la UTAUT}

La investigación para poder explicar la relación entre la aceptación y la adopción de la tecnología, requería integrar la intención de las personas para usarlas, por lo cual se siguieron estudiando diferentes aspectos de la conducta hasta llegar a la Teoría unificada de la aceptación y el uso de la tecnología (UTAUT) que es la que actualmente tiene mayor difusión.

$\mathrm{Su}$ antecedente directo es el Modelo de Aceptación Tecnológica (TAM) presentado por Davis en 1986, el cual se adapta de la Teoría de Acción Razonado para interpretar la aceptación de los usuarios hacia los sistemas tecnológicos de información, agregando dos creencias específicas: utilidad y facilidad. sin embargo, y de acuerdo a Davis (1989), aunque estas creencias influían en el uso de la tecnología, aún se debían incluir variables externas.

Al dejar de lado la influencia social, la aceptación de la tecnología estaba limitada a espacios específicos (por ejemplo, solo para el trabajo), por lo cual se necesitaba un instrumento que incluyera variables externas para obtener resultados más consistentes como la motivación (Lin, Fofanah \& Liand, 2011), dado que la aceptación y uso de las herramientas tecnológicas no solo se refiere al uso que le da el cliente, sino también para satisfacer sus necesidades emocionales (Taherdoost, 2018).

Buscando resolver las debilidades de los modelos anteriores, la investigación al respecto dio como resultado la versión final de la Teoría unificada de la aceptación y el uso de la tecnología (UTAUT), desarrollado por Venkatesh, Morris, Davis, \& Davis en el 2003.
Este modelo fue creado a partir de ocho modelos teóricos previos derivados de las creencias propuestas por Davis (1989), y la influencia que tienen sobre la intención de comportamiento. Estos modelos son los siguientes:

El Modelo de la Acción Razonada de Fishbein y Ajzen (1975), la Teoría de la Conducta Planeada de Ajzen, (1991), el Modelo Motivacional de Vallerand (1997), el Modelo de Aceptación a la Tecnología de Davis (1989), Modelo de Utilización de la PC de Thompson (1991), la Teoría Social Cognitiva de Bandura (1986), la Teoría de la Difusión de la Innovación planteada por Rogers en 1962 (Santander Ramírez \& Hormazábal Saavedra, 2015), un modelo híbrido entre la Teoría de la Conducta Planeada y el Modelo de Aceptación a la Tecnología de Taylor \& Todd en 1995 (Xie, 2017).

La teoría UTAUT identifico cuatro antecedentes que se repetían en los modelos anteriores, por lo cual se adaptaron 14 constructos iniciales. Los constructos significativos eran sobre expectativa de esfuerzo y desempeño, influencia social y las condiciones facilitadoras.

Las expectativas de esfuerzo y desempeño se relacionan con la facilidad que el usuario (en este caso, el docente) al esperar que la integración de la tecnología, le dará un resultado especifico. Por otro lado, la influencia social se refiere a lo que el resto espera de ellos ante el uso de la tecnología.

Al respecto, Venkatesh y col. (2003) encontraron que para facilitar esta expectativa e influencia, la condición estará relacionada por la experiencia y la edad del individuo, de tal manera que se integraron como variables moderadoras la experiencia, la edad, el género y se integró la voluntariedad de uso.

Estos modelos y teorías que explican la aceptación y uso de la tecnología, tienen un punto en común: un fuerte componente social. Estos modelos sugieren que la experiencia juega un papel decisivo en la toma de decisiones en lo que a su comportamiento se refiere. 
Figura 2. Adaptación del Modelo UTAUT

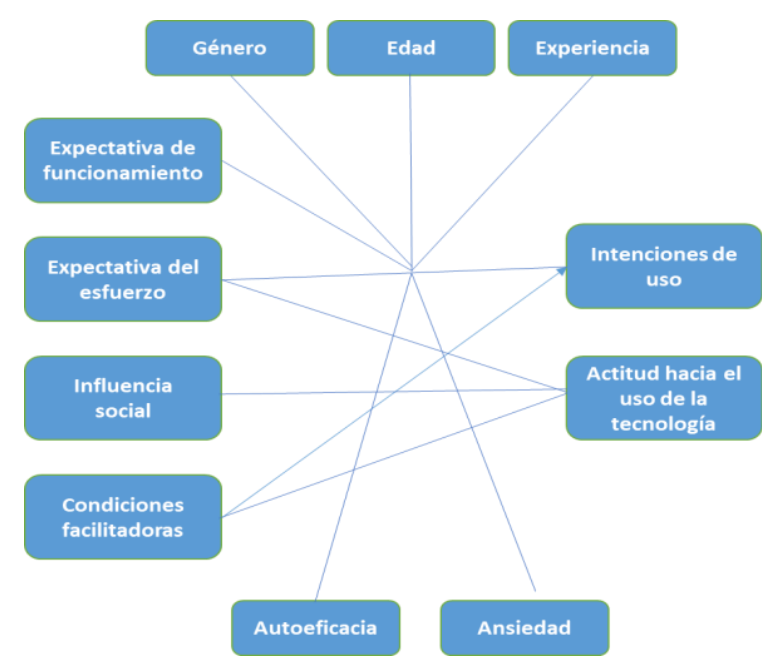

Fuente: Venkatesh y col.;2003, adaptado por Alegre-Cuevas, 2021

La figura 2 nos muestra la adaptación del Modelo UTAUT a partir de los constructos presentados por Venkatesh et al (2003), sin embargo, en esta adaptación presentada, se destacan las experiencias previas y la influencia que podrían tener ante la aceptación y uso de la tecnología (Michel Madera \& Torres Nabel, 2012), integrando además variables como la motivación, la autoeficacia y más recientemente, la innovación.

Al respecto, Tapasco \& Giraldo (2017) encontraron que las tecnologías, su aceptación y uso se ha popularizado en los docentes universitarios, encontrando incluso diferencias significativas entre docentes del sector público y privado, sin embargo, no reportaron diferencias significativas en variables como la experiencia, el sexo, ó la escolaridad. Si estos hallazgos anunciaban la inminente aceptación y uso de tecnologías en el aula, la contingencia de salud derivada de la pandemia por Covid 19 la precipitó.

\section{Metodología}

Utilizando una metodología de corte cuantitativo, con un diseño no experimental de alcance

descriptivo exploratorio, se obtuvieron 100 repuestas al instrumento de la Teoría Unificada de Aceptación y Uso de la Tecnología (UTAUT) de Venkatesh et al., (2003), utilizando la versión adaptada de Michel Madera y Torres Nabel (2012), el cual consta de 31 reactivos tipo Likert con respuestas de 7 puntos que van desde el 1: Nada de acuerdo hasta el 7: Totalmente de acuerdo. Está conformado por 8 sub-escalas: Expectativa del funcionamiento (ítems 1 al 4), Expectativa del esfuerzo (ítems 5 al 8), Actitud hacia el uso de la tecnología (ítems 9 al 12), Influencia social (ítems 13 al 16), Condiciones facilitadoras (ítems 17 al 20), Autoeficacia (ítems 21 al 24), Ansiedad (ítems 25 al 28), Intención de uso (ítems 29 al 31).

Las sub-escalas del UTAUT (Venkatesh et al., 2003) toman en cuenta los siguientes constructos: la sub-escala Expectativa de funcionamiento, considera si la persona cree si la tecnología servirá de apoyo para su desempeño laboral, en este caso en el aula, la sub-escala Expectativa del esfuerzo, está asociada a qué tan sencillo de utilizar es el sistema, la sub-escala Actitud hacia el uso de la tecnología considera lo que las ideas que la persona construye en torno al uso de la misma y cómo influyen en su comportamiento, la sub-escala Influencia social se asocia a lo que la persona percibe de su grupo o pares sobre la importancia del uso de la tecnología, la sub-escala Condiciones facilitadoras toma en cuenta las creencias que tiene la persona acerca de las condiciones tecnológicas y de infraestructura de la organización, en éste caso, de la Institución Educativa, la sub-escala Autoeficacia toma en 
cuenta lo que la persona considera que es capaz de lograr, la sub-escala Ansiedad se refiere a la respuesta que la persona, de manera natural, presenta ante condiciones de estrés, y la subescala Intención de uso considera que tan probable la persona utilizará la tecnología, en el caso de los docentes, en el entorno educativo.

El instrumento fue contestado por 100 docentes de una universidad pública del noreste de México, 49 hombres y 51 mujeres, con una edad promedio de 45 años, con un rango de edad entre 33 y 73 años. Se consideraron 4 categorías de experiencia docente asociadas a la antigüedad del participante en el puesto: de 0 a 5 años, de 6 a 10 años, de 10 a 20 años, y de más de 20 años de docencia.

Los participantes respondieron el UTAUT de manera voluntaria, a través de un formulario digital compartido en grupos de docentes de dicha universidad.
Se utilizó el software PSPP para el procesamiento de los datos y los análisis descriptivos e inferenciales pertinentes (análisis descriptivos, $t$ de student para muestras independientes para establecer diferencias entre sexos y ANOVA de una vía para establecer diferencias entre experiencia docente). Para facilitar la comprensión de los resultados se han convertido todos los puntajes a una escala del 1 al 100.

\section{Resultados}

El objetivo central del presente trabajo fue conocer la aceptación y uso de la tecnología en docentes universitarios, a través del UTAUT.

Como puede observarse en la Tabla 1, la mayoría de la muestra se sitúa en la categoría de experiencia docente de entre 10 a 20 años y los de 20 años y más, por lo que no se pueden considerar como docentes "novatos", es decir, que esta muestra ya tiene experiencia y práctica docente.

Tabla 1: Experiencia docente

\begin{tabular}{ll}
\hline Experiencia docente & Participantes \\
\hline 0 a 5 años & 18 \\
6 a 10 años & 15 \\
10 a 20 años & 33 \\
20 años y más & 34 \\
\hline
\end{tabular}

Fuente: Elaboración Propia

La tabla 2 agrupa los resultados descriptivos de cada sub-escala. Analizando lo anterior se muestra el puntaje superior a 80 en cada una de las sub-escalas, a excepción de ansiedad, ya que esta reporta una media de 30.82, y a diferencia de los demás puntajes, se espera que esta sub-escala obtenga valores mínimos, ya que reporta el grado de ansiedad que los docentes presentan hacia el uso de la tecnología.

Tabla 2: Resultados Descriptivos

\begin{tabular}{lllllllll}
\hline & $\begin{array}{c}\text { Expectativa } \\
\text { de } \\
\text { funcionamie } \\
\text { nto }\end{array}$ & $\begin{array}{c}\text { Expectativa } \\
\text { del esfuerzo }\end{array}$ & $\begin{array}{c}\text { Actitud } \\
\text { hacia el } \\
\text { uso de la } \\
\text { tecnología }\end{array}$ & $\begin{array}{c}\text { Influencia } \\
\text { Social }\end{array}$ & $\begin{array}{c}\text { Condiciones } \\
\text { facilitadoras }\end{array}$ & Autoeficacia & $\begin{array}{c}\text { Ansiedad } \\
\text { Intención } \\
\text { de uso }\end{array}$ \\
\hline Media & 92.46 & 92.11 & 86.75 & 86.25 & 90.46 & 83.54 & 30.82 & 93.71 \\
Des. Est. & 11.03 & 10.04 & 16.89 & 14.41 & 12.73 & 19.82 & 23.23 & 12.40 \\
\hline
\end{tabular}

Para conocer si había diferencia según la experiencia docente de los participantes, se realizó un análisis ANOVA de una vía, obteniendo al inicio los datos pertenecientes a las medias de cada grupo, y estos datos los podemos observar en la Tabla 3.

Tabla 3: Medias por grupo de experiencia docente 


\begin{tabular}{|c|c|c|c|c|c|c|c|c|}
\hline $\begin{array}{l}\text { Grupo según } \\
\text { experiencia } \\
\text { docente en } \\
\text { años }\end{array}$ & $\begin{array}{c}\text { Expectativa de } \\
\text { Funcionamiento }\end{array}$ & $\begin{array}{l}\text { Expectativa } \\
\text { de esfuerzo }\end{array}$ & $\begin{array}{c}\text { Actitud } \\
\text { hacia la } \\
\text { tecnología }\end{array}$ & $\begin{array}{l}\text { Influencia } \\
\text { social }\end{array}$ & $\begin{array}{c}\text { Conductas } \\
\text { facilitadoras }\end{array}$ & Autoeficacia & Ansiedad & $\begin{array}{c}\text { Intención } \\
\text { de uso }\end{array}$ \\
\hline 0 a 5 & 93.65 & 92.86 & 84.92 & 86.11 & 92.46 & 80.95 & 20.83 & 94.97 \\
\hline 6 a 10 & 93.81 & 94.05 & 87.62 & 88.33 & 90.95 & 82.38 & 20.95 & 92.70 \\
\hline 10 a 20 & 90.91 & 90.58 & 83.33 & 84.74 & 87.55 & 83.23 & 32.68 & 91.49 \\
\hline$>20$ & 92.75 & 92.33 & 90.65 & 86.87 & 92.02 & 85.71 & 38.66 & 95.66 \\
\hline
\end{tabular}

Al revisar los datos de la tabla 3 se observa, de primera intención, que las medias de la sub-escala Ansiedad presentan variaciones más pronunciadas.
Se procedió a realizar una comparación por sub-escala del UTAUT utilizando un análisis de varianza de una vía (ANOVA) entre grupos (experiencia docente), obteniendo lo siguiente:

Tabla 4. Resultados ANOVA

\begin{tabular}{lcccc}
\hline Sub-escala & Suma de Cuadrados & gl & F & p \\
\hline Expectativa del funcionamiento & 135.12 & 3 & .36 & .780 \\
Expectativa de esfuerzo & 144.84 & 3 & .47 & .703 \\
Actitud hacia la tecnología & 974.27 & 3 & 1.14 & .336 \\
Influencia social & 153.73 & 3 & .24 & .868 \\
Conductas facilitadoras & 436.72 & 3 & .90 & .447 \\
Autoeficacia & 304.68 & 3 & .25 & .859 \\
Ansiedad & 5457.82 & 3 & 3.64 & .015 \\
Intención de uso & 336.32 & 3 & .72 & .541 \\
\hline
\end{tabular}

Fuente: Elaboración Propia

Solamente la sub-escala Ansiedad reporta una diferencia significativa según la experiencia docente, con un valor $\mathrm{F}(3,96)=$ $3.64, \mathrm{p}=.015$, por lo que se ejecuta una prueba Post hoc de Tukey para revisar en cuáles grupos se presenta esta diferencia (experiencia docente), encontrándolas entre los grupos de 0 a 5 años y de 20 y más años de experiencia docente $(\mathrm{p}=$ .037)
Para conocer si hay diferencias entre hombres y mujeres, se realizó una prueba $t$ de Student para muestras independientes, reportando diferencia significativa en la sub-escala Autoeficacia. Los resultados completos se pueden observar en la tabla 5

Tabla 5: Resultados de t de Student para diferencia entre hombres y mujeres

\begin{tabular}{llll}
\hline Sub-escala & $\mathrm{t}$ & $\mathrm{gl}$ & $\mathrm{p}$ \\
\hline Expectativa del funcionamiento & -.93 & 98 & .354 \\
Expectativa de esfuerzo & -.95 & 98 & .347 \\
Actitud hacia la tecnología & .09 & 98 & .926 \\
Influencia social & .17 & 98 & .869 \\
Conductas facilitadoras & .29 & 98 & .773 \\
Autoeficacia & 2.09 & 98 & .039 \\
Ansiedad & .06 & 98 & .954 \\
Intención de uso & -.59 & .98 & .558 \\
\hline
\end{tabular}

\section{Conclusiones}

Los cambios en el entorno académico derivados de la contingencia de salud por Covid 19 han puesto a los docentes universitarios ante el reto de adaptar y/o migrar los contenidos educativos a una modalidad en línea, lo anterior ha puesto a prueba además a las Instituciones de Educación
Superior en aspectos como infraestructura capacitación de los cuerpos docentes.

En el presente proyecto se pretendía, a través de un estudio exploratorio, conocer la aceptación y el uso de la tecnología de los docentes universitarios desde la Teoría unificada de la aceptación y el uso de la tecnología, 
propuesta por Venkatesh et al., (2003) a través del instrumento UTAUT.

En los resultados obtenidos se puede observar que los puntajes descriptivos de todas las sub-escalas, a excepción de Ansiedad, son superiores al tercer cuartil, lo que podemos interpretar como una aceptación positiva en el uso de la tecnología, el soporte social e institucional, además de aspectos como la autoeficacia, esto puede deberse a los planes y estrategias que la Institución Educativa puso en marcha para afrontar estos desafíos.

En lo que respecta a la sub-escala de Ansiedad, entre menor es el puntaje, menor el grado de la variable, por lo que puntajes abajo del primer cuartil son deseables, sin embargo, se encontraron valores por encima de este nivel, pero sin llegar al segundo cuartil, lo que sugiere un estudio a profundidad del fenómeno para brindar el apoyo necesario a los docentes. Ya sea en capacitación o acompañamiento.

Es de utilidad subrayar que también se encontraron diferencias significativas en la sub- escala Ansiedad al comparar los grupos de experiencia docente, siendo el grupo de docentes con 20 años o más frente a aulas los que reportaron valores más altos, esto puede deberse a preconcepciones de dificultad en el uso de las tecnologías.

Se encontraron diferencias significativas entre hombres y mujeres en la sub-escala Autoeficacia, Del Prete \& Cabero (2020) no hallaron diferencias en el uso de tecnologías en ambientes de aprendizaje, sin embargo, Giménez Lozano \& Morales Rodriguez (2019) si observaron diferencias significativas en autoeficacia en profesores universitarios hombres y mujeres.

Aunque el alcance del presente estudio es exploratorio, deja claro la necesidad de seguir investigando el fenómeno, poniendo particular atención a variables como la experiencia docente, y otras no contempladas como la disciplina del docente, así como las características de la Institución de Educación Superior. 


\section{Referencias}

Alshammari, S.H. \& Rosli, M.S. (2020). A Review of Technology Acceptance Models and Theories. Innovative Teaching and Learning Journal (ITLJ). 4(2). 12-22.

Ajzen, I. (1991). The theory of planned behavior. Organizational Behavior and Human Decision Processes, 50(2), 179-211. https://doi.org/10.1016/0749-5978(91)90020-T

ANUIES (2020). Plataformas y recursos digitales ante la contingencia de la COVID-19. Recuperado de: https://recursosdigitales.anuies.mx/acciones-de-contingencia-y-continuidad-de-las-universidades/

Bandura, A. (1986). Social Foundations of Thought and Action: A Social Cognitive Theory. Englewood Cliffs, NJ: Prentice-Hall.

Cacante Caballero, E. (2020). Análisis De Aceptación Y El Uso De La Tecnología Por Medio De La Metodología UTAUT, En La Facultad De Administración De Empresas En La Universidad Autónoma Latinoamericana (UALA). Conocimiento Semilla, 5 (junio), 77-90. https://publicaciones.unaula.edu.co/index.php/semilla/article/view/726

Del Prete, A., \& Cabero Almenara, J. (2020). El uso del Ambiente Virtual de Aprendizaje entre el profesorado de educación superior: un análisis de género. Revista de Educación a Distancia (RED), 20(62). https://doi.org/10.6018/red.400061

Davis, F.D. (1989). Perceived usefulnessusefulnes, perceived ease of use, and user acceptance of information technology. MIS Quarterly, 13 (3), 319-340. https://doi.org/10.2307/249008

El-Besomey, Dina Ali Mohamed. (2020) The Contemporary Vision of Universal Strategic Planning for Facing (COVID-19) Crisis in the Field of Higher Education Via Virtual Learning - Training, European Journal of Education. 3(2). https://EconPapers.repec.org/RePEc:eur:ejedjr:97.

Fishbein, M., y Ajzen, I. (1975). Belief, attitude, intention, and behavior: An introduction to theory and research. Reading, Addison-Wesley Pub. Co.

Giménez Lozano, J. M.; Morales Rodríguez, F. M. (2019). Relación Entre Las Creencias De Autoeficacia Y Los Niveles De Inteligencia Emocional En Docentes Universitarios. International Journal of Developmental and Educational Psychology, 1 (1) 143-155.

Marinoni, Giorgio, Van't Land, Hilligje, \& Jensen, Trine. (2020). The Impact of COVID-19 on Higher Education Around the World IAU Global Survey Report. Recuperado de: https://www.iauaiu.net/IMG/pdf/iau_covid19_and_he_survey_report_final_may_2020.pdf

Michel, M. \& Torres, L. (2012) Estudio de traducción y confiabilidad del instrumento de la Teoría Unificada de la Aceptación y Uso de la Tecnología (UTAUT). Apertura (17), 96-105.

Momani, A. M. (2018). Adoption of Acceptance Theory and Social Commerce Use (TAUSC Model) for Consumer Behaviour. Al-Madinah International University

Momani, A. (2020). The unified theory of acceptance and use of technology: a new approach in technology acceptance. International Journal of Sociotechnology and Knowledge Development. Jun. DOI: 10.4018/IJSKD.2020070105

OMS (2020) COVID-19: Cronología de la actuación de la OMS. Recuperado de: https://www.who.int/es/news/item/27-04-2020-who-timeline---covid-19

Oye, N.D., Iahad, N., y AbRahim, N.Z. (2014). The history of UTAUT model and its impact on ICT acceptance and usage by academicians. Educ Inf Technol, (19), 251-270. https://doi.org/10.1007/s10639-012-9189-9

Pillai, R. \& Sivathanu, B. (2020). An empirical study on the online learning experience of MOOCs: Indian students' perspective". International Journal of Educational Management. 34 (3), 586-609. https://doi.org/10.1108/IJEM-01-2019-0025

Sánchez, J., Olmos, S., y García, F. (2017). Motivación e innovación: Aceptación de tecnologías móviles en los maestros en formación. Revista Iberoamericana de Educación a Distancia, 20 (2), 273-292. http://dx.doi.org/10.5944/ried.20.2.17700

Santander Ramírez V., \& Hormazábal Saavedra P. (2015). Difusión de la Innovación Curricular por Competencias. Revista Académica de la Universidad Católica del Maule (48), 29-53. 
Taherdoost, H. (2018). A review of technology acceptance and adoption models and theories. In 11th International Conference Interdisciplinary in Engineering. Procedia Manufacturing. 22. 960-967. 10.1016/j.promfg.2018.03.137

Tapasco, Omar A, \& Giraldo, Jaime A. (2017). Comparative Study about Perception and use of ICT among Teachers of Public and Private Universities. Formación universitaria, 10(2), 03-12. https://dx.doi.org/10.4067/S0718-50062017000200002

Thompson, R. L., Higgins, C.A., \& Howell, J. M. (1991). Personal Computing: Toward a Conceptual Model of Utilization. MIS Quarterly, 15(1), 1214-143.

Vallerand, R. J. (1997). Toward a hierarchical model of intrinsic and extrinsic motivation. In M. P. Zanna (Ed.), Advances in experimental social psychology: vol. 29 (pp. 271-360). San Diego: Academic Press.

Venkatesh, V., Morris, M.G., Davis, F.D., y Davis, G.B. (2003). User acceptance of information technology: towards a unified view. MIS Quarterly, 27(3), 425-478.

Williams, M., Rana, N.P., y Dwivedi, Y.K.. (2015). "The unified theory of acceptance and use of technology (UTAUT): a literature review", Journal of Enterprise Information Management, (28) 3, 443-488. https://doi.org/10.1108/JEIM-09-2014-0088

Xie, Q., Song, W., Peng, X., \& Shabbir, M. (2017). Predictors for e-government adoption: integrating TAM, TPB, trust and perceived risk. The Electronic Library, 35(1), 2-20. doi:10.1108/el-08-2015-0141 